PROCEEDINGS OF THE

AMERICAN MATHEMATICAL SOCIETY

Volume 130, Number 9, Pages 2625-2630

S 0002-9939(02)06361-X

Article electronically published on February 12, 2002

\title{
ON THE PERIODIC POINTS OF FUNCTIONS ON A MANIFOLD
}

\author{
CHUNG-WU HO
}

(Communicated by Alan Dow)

\begin{abstract}
In a conference on fixed point theory, B. Halpern of Indiana University considered the problem of reducing the number of periodic points of a map by homotopy. He also asked whether the number of periodic points of a function could be increased by a homotopy. In this paper, we will show that for any map on a closed manifold, an arbitrarily small perturbation can always create infinitely many periodic points of arbitrarily high periods.
\end{abstract}

\section{The MAIN REsUlts}

Let $X$ be a topological space and $f: X \rightarrow X$ a continuous map. A point $x \in X$ is said to be a periodic point of period $p$ if $f^{p}(x)=x$ but $f^{k}(x) \neq x$ for each positive integer $k<p$. In a conference on fixed point theory in Bloomington, Indiana in 1980, B. Halpern considered the problem of reducing the number of periodic points of a continuous function of a closed manifold by homotopy [2]. He also raised the problem as how to increase the number of periodic points of such a map by homotopy. We will study this problem in the present paper. We first consider the case for the identity map on a manifold. We will show that for an arbitrary manifold of dimension greater than 1, an arbitrarily small local perturbation of the identity map by a homotopy may generate uncountably many periodic points of period $p$ for each positive integer $p$. Specifically, we have the following.

Theorem 1.1. Let $M$ be an arbitrary manifold of dimension $\geq 1$ with a possibly nonempty boundary. For any nonempty open set $U$ of $M$, there exists a map $g$ : $M \rightarrow M$ such that:

(1) $g$ agrees with the identity map id of $M$ on the set $M-U$;

(2) $g$ is homotopic to id under a homotopy that is pointwise fixed on $M-U$; and

(3) for each positive integer $n$, the map $g$ has a periodic point of period $n$.

If $\operatorname{dim}(M)>1$, we may find a map $g: M \rightarrow M$ satisfying (1) and (2) above and possessing uncountably many periodic points for each period $n$.

If the identity map of $M$ is replaced by an arbitrary map $f$ of $M$ into $M$, can we still generate a large number of periodic points by a small perturbation of $f$ ? The answer is clearly no if we do not impose any restriction on the manifold $M$. Consider

Received by the editors February 9, 1999 and, in revised form, April 1, 2001.

2000 Mathematics Subject Classification. Primary 37C25; Secondary 54H25, 58C30.

Key words and phrases. Manifolds, periodic points, homotopy, digraphs.

(C)2002 American Mathematical Society 
the real valued function $f$ on the real line defined as the translation $f(x)=x+1$. Any map $g$ on the real line sufficiently close to $f$ (in the Whitney $C^{0}$ topology, say) will not have any periodic point either. But if $M$ is compact, the situation is quite different.

Theorem 1.2. Let $M$ be a compact manifold of dimension $\geq 1$ with possibly nonempty boundary. Select a metric $d$ on $M$. For each continuous $f: M \rightarrow M$ and each $\epsilon>0$, there exists a map $g: M \rightarrow M$ such that:

(1) for every $x$ in $M, d(f(x), g(x))<\epsilon$;

(2) the map $g$ is homotopic to $f$ under an $\epsilon$-homotopy, i.e., a homotopy that will not move any point a distance more than $\epsilon$; and

(3) for each positive integer $n$, the map $g$ has infinitely many periodic points of periods greater than $n$ (again, the word "infinitely many" may be replaced by "uncountably many" if $\operatorname{dim}(M)>1)$.

In a private communication, Professor Halpern showed me a way of modifying a map by homotopy to create a large number of periodic points by means of the Tietze extension theorem and the homotopy extension theorem. However, unlike our results of making small perturbations, his construction requires global changes of the given map. The proofs of our two theorems are based on ideas developed in studying the dynamical properties of maps of the real line ([1], 3], 4], 6], and 8]). These ideas will be described in Section 2 The proofs of the theorems will be given in Section 3 ,

\section{Preliminaries on interval DigraphS}

Let $\left\{I_{1}, I_{2}, \cdots, I_{k}\right\}$ be a finite collection of compact intervals of the real line $\mathbb{R}$ such that the interiors of $I_{1}, I_{2}, \cdots, I_{k}$ are pointwise disjoint. Let $f: \mathbb{R} \rightarrow \mathbb{R}$ be a continuous function. We may define a digraph (i.e., a directed graph) associated with $f$ and the intervals $\left\{I_{1}, I_{2}, \cdots, I_{k}\right\}$ as follows. The digraph has a vertex for each interval $I_{i}$ (again denoted by $I_{i}$ ), and a directed arc is drawn from the vertex $I_{i}$ to the vertex $I_{j}$ if and only if $f\left(I_{i}\right) \supset I_{j}$. A cycle in this digraph is a sequence of directed arcs such that the terminal point of each arc coincides with the initial point of the next arc, and the terminal point of the last arc coincides with the initial

point of the first arc. Using the digraphs, we may predict the existence of periodic points of $f$ by means of the following lemma (cf. 8], pp. 7-8).

Lemma 2.1. Let $I_{1}, I_{2}, \cdots, I_{k}$ be compact intervals of the real line $\mathbb{R}$, and let $f$ be a continuous function of $\mathbb{R}$. If the associated digraph contains a cycle $J_{1} J_{2} \cdots J_{n}$ for some vertices $J_{1}, J_{2}, \cdots, J_{n}$ (not necessarily distinct) of the digraph, then there exists a point $p \in J_{1}$ such that $f^{i}(p) \in J_{i+1}$ for each $i=1,2, \cdots, n-1$ and $f^{n}(p)=p$.

Using this lemma, we now show that an arbitrary perturbation of the identity map of the real line may yield a map with many periodic points.

Lemma 2.2. For any point $x_{0} \in \mathbb{R}$ and $\epsilon>0$, there exists a continuous map $e: \mathbb{R} \rightarrow \mathbb{R}$ such that:

(1) e agrees with the identity map id for all $x \notin\left(x_{0}-\epsilon, x_{0}+\epsilon\right)$;

(2) $|e(x)-x|<\epsilon$; and

(3) for each positive integer $k$, the map e has a periodic point of period $k$. 
Proof. Let $x_{0}$ in $\mathbb{R}$ and $\epsilon>0$ be given. Choose $x_{1}$ and $x_{2}$ such that

$$
x_{0}-\frac{\epsilon}{2}<x_{1}<x_{0}<x_{2}<x_{0}+\frac{\epsilon}{2}
$$

Then, choose a map $e: \mathbb{R} \rightarrow \mathbb{R}$ with the following properties:

(1) $e(x)=x$ for all $x \leq x_{0}-\epsilon$ and for all $x \geq x_{2}$, and

(2) $e\left(x_{0}\right)=x_{1}$ and $e\left(x_{1}\right)=x_{2}$

such that $e$ does not move any point by a distance more than $\epsilon$. We contend that $e$ has periodic points of all periods. Clearly, $e$ has many fixed points. To see that $e$ has periodic points of all the periods greater than 1 , let $I_{1}$ and $I_{2}$ be the intervals $\left[x_{1}, x_{0}\right]$ and $\left[x_{0}, x_{2}\right]$, respectively. From the definition of $e$, it is easily seen that the associated digraph for $e$ contains a directed arc from $I_{1}$ to $I_{2}$, a directed arc from $I_{2}$ to $I_{1}$, and a loop at each of the vertices $I_{1}$ and $I_{2}$ :
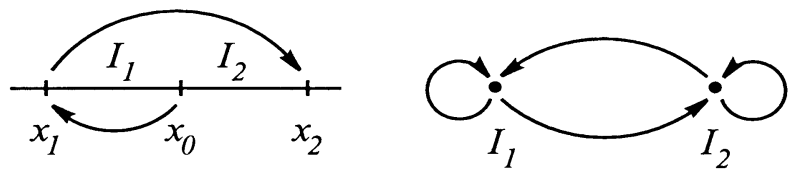

Now, for any $k \geq 2$, consider the $k$-cycle obtained by tracing the graph from the vertex $I_{1}$ to $I_{2}$ and back to $I_{1}$ and followed by tracing the loop at $I_{1}$ for $k-2$ times. This cycle produces a point $p \in I_{1}$ with $f^{k}(p)=p$ as described by [2.1. We now show that $p$ is indeed of period $k$, i.e., $p$ is not of a lower period than $k$. First observe that $p$ and each of its images always lie in the interior of either $I_{1}$ or $I_{2}$. Since the interior of $I_{1}$ and $I_{2}$ are disjoint, if $p$ is of a lower period, the $k$-cycle will have to be a repetition of a single shorter unit. But the $k$-cycle described above is not such a repetition. This proves the lemma.

Lemma 2.3. Let $n \geq 2$ be a given integer. Suppose $B(0 ; r)$ is the $n$-dimensional euclidean ball centered at the origin and having a radius $r>0$. For each $\epsilon>0$, there exists a continuous map $\bar{e}: B(0 ; r) \rightarrow B(0 ; r)$ such that

(1) $\|\bar{e}(x)-x\|<\epsilon$, and

(2) for each positive integer $k$ the map $\bar{e}$ has uncountably many points of period $k$.

Proof. Let $S^{n}(0 ; r)$ be the $n$-dimensional sphere of radius $r$ centered at the origin of $\mathbb{R}^{n+1}$. Applying the technique of 2.2 to a small interval of the circle $S^{1}(0 ; r)$, we may get a map $g: S^{1}(0 ; r) \rightarrow S^{1}(0 ; r)$ which does not move any point of the circle by a distance more than $\epsilon$ and which possesses periodic points of all the possible periods. Taking successive suspensions of both $g$ and the space, we can get a similar map $h: S^{n-1}(0 ; r) \rightarrow S^{n-1}(0 ; r)$. Now, a radial extension $\bar{e}(t x)=t \cdot h(x)$ for all $x \in S^{n-1}(0 ; r)$ and $0 \leq t \leq 1$ produces a desired map $\bar{e}$.

\section{PROOF OF THE THEOREMS}

3.1. Proof of Theorem 1.1. Let $M$ be an arbitrary manifold of dimension $\geq 1$, and $U$ a nonempty open set of $M$. Choose a coordinate neighborhood $(V, \phi)$ of $M$ such that $V \subset U$ and $\phi(V)$ contains a euclidean ball $B(0 ; 2 t)$ centered at the origin of $\mathbb{R}^{n}$ and having a radius of $2 t$ for some positive number $t$. 
Fix a positive number $s$ less than $t / 3$ and choose a continuous real valued function $h$ of the real line with the properties that

(1) $0 \leq h(x) \leq 1$ for all $x$ in $\mathbb{R}$,

(2) $h(x)=1$ for all $x \leq t+s$ and

(3) $h(x)=0$ for all $x \geq 2 t-s$.

Now, define a function $G: B(0 ; 2 t) \rightarrow B(0 ; 2 t)$ by

$$
G(x)=h(\|x\|) e(x)+(1-h(\|x\|) x
$$

for all $x$ in $B(0 ; 2 t)$, where $e: B(0 ; 2 t) \rightarrow B(0 ; 2 t)$ is the function given in 2.2 (with $x_{0}=0$ and $\epsilon=t$ ) if $\operatorname{dim}(M)=1$, and the function $\bar{e}$ of 2.3 (with $r=2 t$ ) if $\operatorname{dim}(M)>1$. Note that for each positive integer $k, G$ has a periodic point of period $k$. If $\operatorname{dim}(M)>1$, the number of periodic points of $G$ of period $k$ is uncountable.

We may now use $G$ to get a map $g: M \rightarrow M$ by letting $W=\phi^{-1}(B(0 ; 2 t))$ and

$$
g(x)=\left\{\begin{array}{cl}
x & \text { if } x \in M-W, \\
\phi^{-1}(G(\phi(x))) & \text { if } x \in W .
\end{array}\right.
$$

The map $g$ satisfies the conclusions of our Theorem 1.1

3.2. Proof of Theorem 1.2. Let $M$ be a compact manifold of dimension $\geq 1$ with possibly a nonempty boundary $\operatorname{Bd}(M)$. Fix on $M$ a metric $d$ which is compatible with the topology of $M$. Consider an arbitrary continuous function $f: M \rightarrow M$ and suppose that a positive number $\epsilon$ is given. Set $\delta=\epsilon / 4$. We will carry out the proof in the following steps:

(1) Applying a $\delta$-homotopy to $f$ if necessary, we may assume that $f$ has a periodic point $p$ of some period. This is seen as follows: choose any point $q$ in $M$, and consider the set of all the iterates $\left\{f^{i}(q) \mid i=1,2, \cdots\right\}$. Since $M$ is compact, this set has an accumulation point. Therefore, there exists an open ball $U$ in $M$ with $\operatorname{diam}(U)<\delta$ such that $U$ contains two of the iterates $f^{j}(q)$ and $f^{k}(q)$. Assuming that $j<k$, we let $p=f^{j}(q)$ and $m=k-j$. Replacing $U$ by a smaller open set if necessary, we may assume that $U$ contains $p$ and $f^{m}(p)$ in the same path component but $U$ does not contain $f^{i}(p)$ for $0<i<m$. Now, choose a map $h$ of $M$ which is pointwise fixed on $M-U$, but within the set $U, h$ slides the point $f^{m}(p)$ onto the point $p$. The map $h \circ f$ is clearly homotopic to $f$ by a homotopy which does not move any point by a distance more than $\delta$, and the point $p$ is now a periodic point of the new map.

(2) Suppose now that $f$ has a periodic point $p$ of period $m$. We now show that, by another $\delta$-homotopy if necessary, we may assume that all the points $p, f(p), \cdots, f^{m-1}(p)$ lie in $\operatorname{Int}(M)$. If any of these points belongs to $\operatorname{Bd}(M)$, we may pick a pathwise connected neighborhood $U_{i}$ with diameter $<\delta / m$ around each point $f^{i}(p)$ for $i=1,2, \cdots, m-1$ such that the $U_{i}$ 's are pairwise disjoint and $f\left(U_{m-1}\right) \subset U_{1}$. Now, since $\operatorname{Bd}(M)$ is nowhere dense in $M$, we may pick a point $q_{i}$ in $\operatorname{Int}(M) \cap U_{i}$ such that $f\left(q_{i-1}\right) \in U_{i}$. For each $i=1,2, \cdots, m-1$, let $h_{i}$ be a deformation which agrees with the identity map outside the set $U_{i}$, and while on the set $U_{i}$, it moves the point $f\left(q_{i-1}\right)$ onto the point $q_{i}$. Finally, let $h_{m}$ be a deformation which is pointwise fixed outside the set $U_{0}$, and which moves the point $f\left(q_{m-1}\right)$ onto $q_{0}$. Since the sets $U_{i}$ are pairwise disjoint, the map $h_{m} \circ \cdots \circ h_{1} \circ f$ will carry each $q_{i-1}$ onto the point $q_{i}$ and carry the point $q_{m-1}$ onto $q_{0}$. This deformation of $f$ 
will then have a periodic point (again, call it $p$ ) of period $m$, whose images all lie in $\operatorname{Int}(M)$.

(3) We now show that, by a third $\delta$-homotopy if necessary, we may assume that $f^{m}$ agrees with the identity map on a neighborhood of $p$. Note that for $f^{m}$ to be locally one to one, $f$ itself will have to be locally one to one on a neighborhood of each of the points $f^{k}(p)$ for $0 \leq k \leq m-1$. Thus, we need to modify the function $f$ in a neighborhood of each of these points.

Let $B(0 ; r)$ be the euclidean ball of radius $r$ centered at the origin. Since each of the points $f^{i}(p)$ lies in $\operatorname{Int}(M)$, we may choose a coordinate system $\left(U_{i}, \psi_{i}\right)$ at the point $f^{i}(p)$ for each $i=1,2, \cdots, m$ as follows: first fix a coordinate system $\left(U_{m}, \psi_{m}\right)$ at the point $f^{m}(p)=p$ in such a way that:

(a) $\operatorname{diam}\left(U_{m}\right)<\delta$, and

(b) $\psi_{m}\left(U_{m}\right)$ contains the euclidean ball $B(0 ; 2)$.

Now, for each positive real number $r \leq 2$, let $U_{m}(r)=\psi_{m}^{-1}(B(0 ; r))$. Then, for each $i=m-1, m-2, \cdots, 2,1$, choose a coordinate system $\left(U_{i}, \psi_{i}\right)$ at the point $f^{i}(p)$ inductively as follows:

(a) the open set $U_{i}$ does not contain any of the points $f^{j}(p)$ for $j \neq i$, and it is disjoint from all the previously chosen sets $U_{m}, U_{m-1}, \cdots, U_{i+1}$,

(b) $\operatorname{diam}\left(U_{i}\right)<\delta$,

(c) $\psi_{i}\left(U_{i}\right)$ contains the euclidean ball $B(0 ; 2)$, and

(d) $f\left(U_{i}\right) \subset U_{i+1}(2)$, where for each $i, U_{i}(r)=\psi_{i}^{-1}(B(0 ; r))$.

Finally, choose a positive real number $a<1$ such that $f\left(U_{m}(2 a)\right) \subset U_{1}(1)$. Now, let $h: \mathbb{R} \rightarrow \mathbb{R}$ be a continuous function with

$$
h(x)= \begin{cases}1 & \text { if }|x| \leq 1 \\ 0 & \text { if }|x| \geq \frac{3}{2}\end{cases}
$$

We may now modify our function $f$ into a map $F: M \rightarrow M$ as follows:

(a) $F(x)=f(x)$ for all $x$ in $M-\bigcup\left\{U_{i} \mid i=1,2, \cdots, m\right\}$.

(b) For each $x$ in $U_{m}$, let

$$
F(x)=\left\{\begin{array}{cl}
\psi_{1}^{-1}\left[h\left(\frac{1}{a}\left\|\psi_{m}(x)\right\|\right) \frac{1}{a} \psi_{m}(x)\right. & \text { if } x \in U_{m}(2 a), \\
\left.+\left(1-h\left(\frac{1}{a}\left\|\psi_{m}(x)\right\|\right) \psi_{1}(f(x))\right)\right] & \\
f(x) & \text { if } x \in U_{m}-U_{m}(2 a) .
\end{array}\right.
$$

Note that $F$ carries the open set $U_{m}(a)$ homeomorphically onto the set $U_{1}(1)$.

(c) Now, for $i=1,2, \cdots, m-2$, define $F$ on the set $U_{i}$ as follows:

$$
F(x)=\left\{\begin{array}{cl}
\psi_{i+1}^{-1}\left[h\left(\left\|\psi_{i}(x)\right\|\right) \psi_{i}(x)\right. & \text { if } x \in U_{i}(2), \\
\left.+\left(1-h\left(\left\|\psi_{i}(x)\right\|\right) \psi_{i+1}(f(x))\right)\right] & \\
f(x) & \text { if } x \in U_{m-1}-U_{m-1}(2) .
\end{array}\right.
$$

(d) Finally, on the set $U_{m-1}$, define

$$
F(x)=\left\{\begin{array}{cc}
\psi_{m}^{-1}\left[h\left(\left\|\psi_{m-1}(x)\right\|\right) a \psi_{m-1}(x)\right. & \text { if } x \in U_{m-1}(2), \\
\left.+\left(1-h\left(\left\|\psi_{m-1}(x)\right\|\right) \psi_{m}(f(x))\right)\right] & \\
f(x) & \text { if } x \in U_{m-1}-U_{m-1}(2) .
\end{array}\right.
$$

Since the sets $U_{i}, i=1,2, \cdots, m$, are pairwise disjoint, $F$ is a well defined continuous map. It is clearly homotopic to $f$ by a homotopy which does not 
move any point to a distance more than $\delta$. Furthermore, for any point $x$ in $U_{m}(a)$

$$
\begin{array}{rlrl}
F^{m}(x) & =F \circ \cdots \circ F(x) & & (m \text { copies of } F) \\
& =F \circ \cdots \circ F\left(\psi_{1}^{-1}\left(\frac{1}{a} \psi_{m}(x)\right)\right) & & (m-1 \text { copies of } F) \\
& =F \circ \cdots \circ F\left(\psi_{2}^{-1} \psi_{1} \psi_{1}^{-1}\left(\frac{1}{a} \psi_{m}(x)\right)\right) & & (m-2 \text { copies of } F) \\
& =\cdots & & \\
& =F\left(\psi_{m-1}^{-1}\left(\frac{1}{a} \psi_{m}(x)\right)\right) & \\
& =\psi_{m}^{-1}\left(a \psi_{m-1}\left(\psi_{m-1}^{-1}\left(\frac{1}{a} \psi_{m}(x)\right)\right)\right) & \\
& =x . &
\end{array}
$$

Thus, $F^{m}$ is the identity map on a neighborhood of $p$.

(4) Now, suppose that $f^{m}$ is the identity map on an open neighborhood $U$ of $p$. Replacing $U$ by a smaller neighborhood if necessary, we may assume that $\operatorname{diam}(U)<\delta$ and that the sets $U, f(U), \cdots, f^{m-1}(U)$ are pairwise disjoint. Now, choose a coordinate system $(V, \phi)$ of $M$ such that $V \subset U$ and $\phi(V)$ contains the euclidean ball $B(0 ; 2 t)$ centered at the origin and having radius $2 t$ for some real number $t$. Let $W=\phi^{-1}(B(0 ; 2 t))$ and define a function $\bar{g}: M \rightarrow M$ the same way as we defined the function $\bar{g}$ in the proof of 1.1 i.e., $\bar{g}$ is the identity map on the set $M-W$, and $\bar{g}$ has a periodic point of period $k$ in $W$ for each positive integer $k$ (and in fact, uncountably many periodic points for each period if $\operatorname{dim}(M)>1$ ). Now, let $g=\bar{g} \circ f$. Since $\bar{g}$ is the identity map outside the open set $U$, and the sets $U, f(U), \cdots, f^{m-1}(U)$ are pairwise disjoint, it follows that $g^{m}\left|U=(\bar{g} \circ f)^{m}\right| U=\bar{g} \circ f^{m}|U=\bar{g}| U$, and hence, for each $k$, the map $g$ will have periodic points of period $m k$. This $g$ satisfies the assertions of 1.2 .

\section{REFERENCES}

1. L. Block, J. Guckenheimer, M. Misiurewicz, and L. Young, Periodic points and topological entropy of one dimensional maps, Lect. Notes Math., 819 (1980), Springer-Verlag, 18-34. MR 82j:58097

2. B. Halpern, The minimal number of periodic points, Abstr. Amer. Math. Soc., 1 (1980), 775-G8, p. 269.

3. C.W. Ho, On Block's condition for simple periodic orbits of functions on an interval, Trans. Amer. Math. Soc., 281 (1984), 827-832. MR 85g:54035

4. C.W. Ho and C. Morris, A graph-theoretical proof of Sharkovsky's theorem on the periodic points of continuous functions, Pacific J. Math., 96 (1981), 361-370. MR 83d:58056

5. M. Hirsch, Differential Topology, Grad. Texts in Math., Vol. 33, Springer-Verlag, 1976. MR 56:6669

6. T. Li and J. Yorke, Period three implies chaos, Amer. Math. Monthly, 82 (1975), 985-992. MR 52:5898

7. J. Munkres, Elementary Differential Topology, Revised Ed., Annals of Math. Studies, 54, Princeton Univ. Press, 1966. MR 33:6637

8. Z. Nitecki, Topological dynamics on the interval, Ergodic Theory and Dynamical System II, Progress in Math., Vol. 21, Ed. by A. Katok, Birkhäuser (1982), 1-73. MR 84g:54051

Department of Mathematics, Southern Illinois University at Edwardsville, Edwardsville, Illinois 62026 - And - Department of Mathematics, Evergreen Valley College, San Jose, California 95135

E-mail address: cho@siue.edu 